\title{
Generation and Characterization of Erythroid Cells from Human Embryonic Stem Cells and Induced Pluripotent Stem Cells: An Overview
}

\author{
Kai-Hsin Chang, ${ }^{1}$ Halvard Bonig, ${ }^{1,2}$ and Thalia Papayannopoulou ${ }^{1}$ \\ ${ }^{1}$ Division of Hematology, Department of Medicine, University of Washington, 1705 NE Pacific, Rm K243, P. O. Box 357710, \\ Seattle, WA 98195-7710, USA \\ ${ }^{2}$ Department of Cellular Therapeutics/Cell Processing, Institute for Transfusion Medicine and Immunohematology, \\ German Red Cross Blood Service, Goethe University, Frankfurt D-60528, Germany
}

Correspondence should be addressed to Thalia Papayannopoulou, thalp@u.washington.edu

Received 12 July 2011; Accepted 29 August 2011

Academic Editor: Anna Rita Migliaccio

Copyright (๑) 2011 Kai-Hsin Chang et al. This is an open access article distributed under the Creative Commons Attribution License, which permits unrestricted use, distribution, and reproduction in any medium, provided the original work is properly cited.

\begin{abstract}
Because of the imbalance in the supply and demand of red blood cells (RBCs), especially for alloimmunized patients or patients with rare blood phenotypes, extensive research has been done to generate therapeutic quantities of mature RBCs from hematopoietic stem cells of various sources, such as bone marrow, peripheral blood, and cord blood. Since human embryonic stem cells (hESCs) and induced pluripotent stem cells (iPSCs) can be maintained indefinitely in vitro, they represent potentially inexhaustible sources of donor-free RBCs. In contrast to other ex vivo stem-cell-derived cellular therapeutics, tumorigenesis is not a concern, as RBCs can be irradiated without marked adverse effects on in vivo function. Here, we provide a comprehensive review of the recent publications relevant to the generation and characterization of hESC- and iPSC-derived erythroid cells and discuss challenges to be met before the eventual realization of clinical usage of these cells.
\end{abstract}

\section{Introduction}

Medical progress, specifically in the fields of hematology/oncology and transplantation surgery, as well as an overall aging population, has led to an ever-increasing demand for erythrocytes for transfusion to currently approximately fifty thousand RBC concentrates per million population per year in countries with a high standard of health care. Currently, the exclusive source for these is volunteer donors, who obviously are subject to the same societal changes as the recipients, that is, they are aging also. Recruitment of new donors from the shrinking pool of eligible individuals is challenging and additionally hampered by ever-increasing restrictions, predominantly recipient-directed exclusion criteria for donors. Perceived lack of safety of blood products also is a highly sensitive issue in the population, particularly since the emergence of HIV in the eighties, as a consequence of which a whole generation of hemophilia patients was infected. The desire to counter these challenges has led to the extensive effort in the generation of red blood cells (RBCs) in vitro from a variety of sources, such as bone marrow, peripheral blood, and cord blood. More recently, utilizing embryonic stem cells (hESCs) and induced pluripotent stem cells (iPSCs) to generate universal donor RBCs has been envisioned $[1,2]$.

hESCs are pluripotent stem cells derived from the inner cell mass of the blastocyst [3], and iPSCs are ESC-like cells generated by reprogramming somatic cells, most often via forced expression of a combination of transcription factors, such as Oct3/4, Nanog, KLF4, c-Myc, LIN28, and SOX2 $[4,5]$. Both hESCs and hiPSCs can be maintained indefinitely in culture and can be induced to undergo differentiation to give rise to any cell types of all three germ layers. These characteristics made them not only a valuable tool for the study of developmental biology, but also a potential source for providing unlimited numbers of cells for cell replacement 
therapies. While our laboratory has previously shown that the then existing hESC lines are not conducive to culturing universal donor RBCs [6], many additional hESC lines have been generated ever since. Furthermore, the recent advances in hiPSCs have partially obviated this obstacle, as exemplified by the generation of hiPSCs from a Bombay individual whose RBCs lack $\mathrm{ABH}$ antigens expression due to the absence of the H gene (FUT1-) and Secretor gene (FUT2-) encoded $\alpha$ 1,2 fucosyltransferase activities [7]. It has been proposed by French researchers that based on their database, as few as 15 hiPSC clones would cover 100\% of the needs of all Caucasian patients with rare blood phenotypes/genotypes in France [2]. In addition, they suggested that one single hiPSC clone would meet $73 \%$ of the needs in alloimmunized patients with sickle cell disease for whom rare cryopreserved RBC units are required [2]. In this review, we summarize the recent development in the in vitro generation and characterization of erythroid cells from either hESCs or iPSCs and point out areas of further investigation needed before their use for clinical purposes.

\section{Methods for the Generation of Erythroid Cells from hESCs and iPSCs}

hESCs and hiPSCs represent renewable, potentially unlimited cell sources, in contrast to the hematopoietic stem cells originating from bone marrow, cord blood, or peripheral blood, that require donors to be constantly secured, for the ex vivo generation of RBCs. The potential charm of autologous hiPSCs in the context of erythrocyte generation is the identical genetic identity between the cells and the recipient for all blood group antigens, since the prevalent clinical problem is the polysensitization against foreign erythrocyte antigens, and these patients are at risk for acquisition of additional antibodies. Several laboratories have established protocols to derive erythroid cells from the hESCs and hiPSCs. While each protocol varies in technical details, they can generally be divided into two main categories: those that coculture stem cells with stromal layers to induce hematopoietic differentiation, and those that culture stem cells in suspension to form embryoid bodies (EBs) (Table 1). One exception is a recent report by Salvagiotto and colleagues who circumvent EBs and stromal coculture by utilizing a matrix protein to generate a 2-dimentioinal culture system to support the generation of hematopoietic cells from hESCs and hiPSCs [25].

2.1. Stromal Coculture. Several types of stromal layers have been reported in the coculture system, including murine bone marrow (BM) cell line S17 $[9,26]$ and OP9 $[8,10,27]$, the yolk sac endothelial cell line C166 [9], murine fetal liverderived stromal line (mFLSC) $[12,13]$, murine aorta-gonadmesonephros (AGM) stromal lines [12], primary murine stromal cells from AGM and FL [12], immortalized human fetal liver hepatocyte line FH-B-hTERT $[1,14,15]$, and primary human stromal cells derived from aorta- AGM, FL, and fetal BM (FBM) [11]. A side-by-side comparison finds that FH-B-hTERT cells are more effective than S17 cells in stimulating CD $34^{+}$cells generation from hESCs [14]. The direct contact with stromal layers increases hematopoietic differentiation efficiency $[11,12,26]$, although it is not an absolute requirement $[11,26]$. In general, when culture media are supplemented with fetal bovine serum (FBS), no additional growth factors are required during coculture $[1,9,12,14,15,26]$. To generate large quantities of relatively pure erythroid cells, often a further purification of CD34 ${ }^{+}$ cells from the coculture population is performed prior to seeding them in media supplemented with growth factors for erythroid expansion $[1,8]$, or the mixture of coculture cells is placed in methylcellulose cultures for hematopoietic colony assays, and then individual erythroid colonies are extracted from semisolid culture for further analysis [13]. While these studies have provided important insights into the erythroid development from hESCs or hiPSCs and have the potential for large-scale production $[1,8,13]$, the utilization of murine cells in coculture by some protocols may make them unsuitable for clinical applications in the future.

2.2. EB Formation. Alternatively, successful hematopoietic induction can be achieved by EB formation, usually by placing clumps of hESCs or hiPSCs in nontissue culture-treated plates, or ultralow attachment plates. It has been reported in mouse EBs that cells undergo self-organization to establish anteroposterior polarity and form a primitive streak-like region via the Wnt signaling pathway [28]. For robust hematopoietic differentiation, good quality EB formation is essential. While $\mathrm{Lu}$ et al. observe that it is essential to use high-quality hESCs with minimal signs of differentiation and uniform stem cell marker expression for EBs formation and for high-efficiency erythroid generation [22], Ungrin and colleagues find that aggregates formed from populations of cells with the highest levels of Oct4 actually exhibit the least stability in terms of EB formation [29]. We find that hESCs or hiPSCs cultured under murine embryonic fibroblast (MEF) feeder-free conditions on matrigel-coated plates with MEFconditioned media or a chemically defined media, such as mTeSR (StemCell Technologies), can also be used for EB formation, although they tend to be more temperamental with less consistent EB formation for unknown reasons (unpublished data). EB formation can be further controlled by plating a defined number of cells in untreated $U$ bottom or V bottom, 96- or 386-well plates, or AggreWells and forcing the formation of aggregates by centrifugation [30, 31]. Because the forced aggregation of a defined number of hESCs or hiPSCs requires the generation of single cell suspensions prior to aggregation, we find that the addition of p160-Rho-associated coiled coil kinase (ROCK) inhibitor Y-27632 before and during the first 24 hours of EB formation is essential for the survival of these stem cells, consistent with previous reports $[29,32]$. However, while forced aggregation leads to uniformly sized EBs, it has been shown that in fact, the traditional suspension culture of placing clumps of hESCs directly into low-attachment plates generates EBs with higher levels of hematopoietic differentiation than forced aggregation or hanging drop culture does [33]. Regardless of the EB formation method employed, it is important 


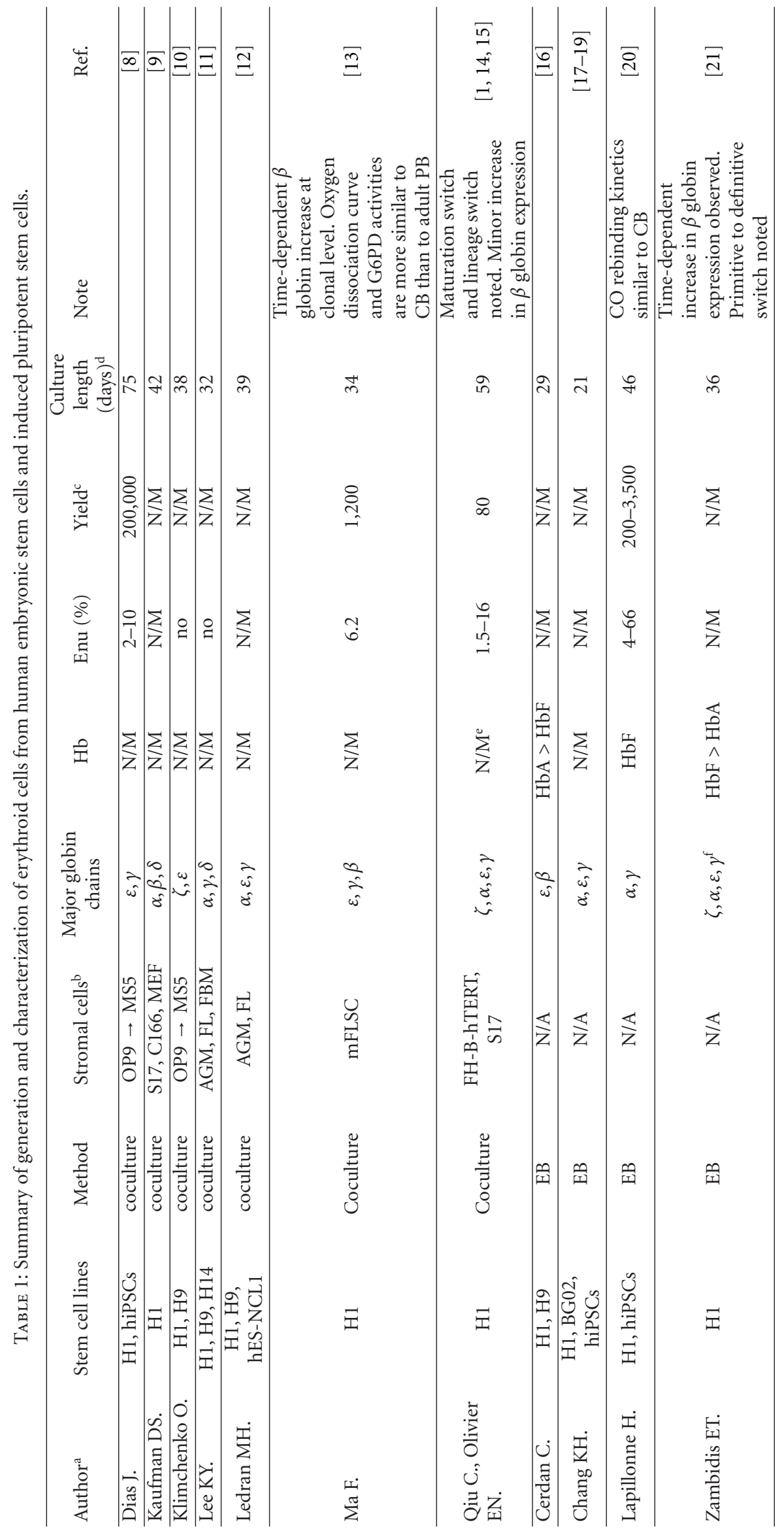




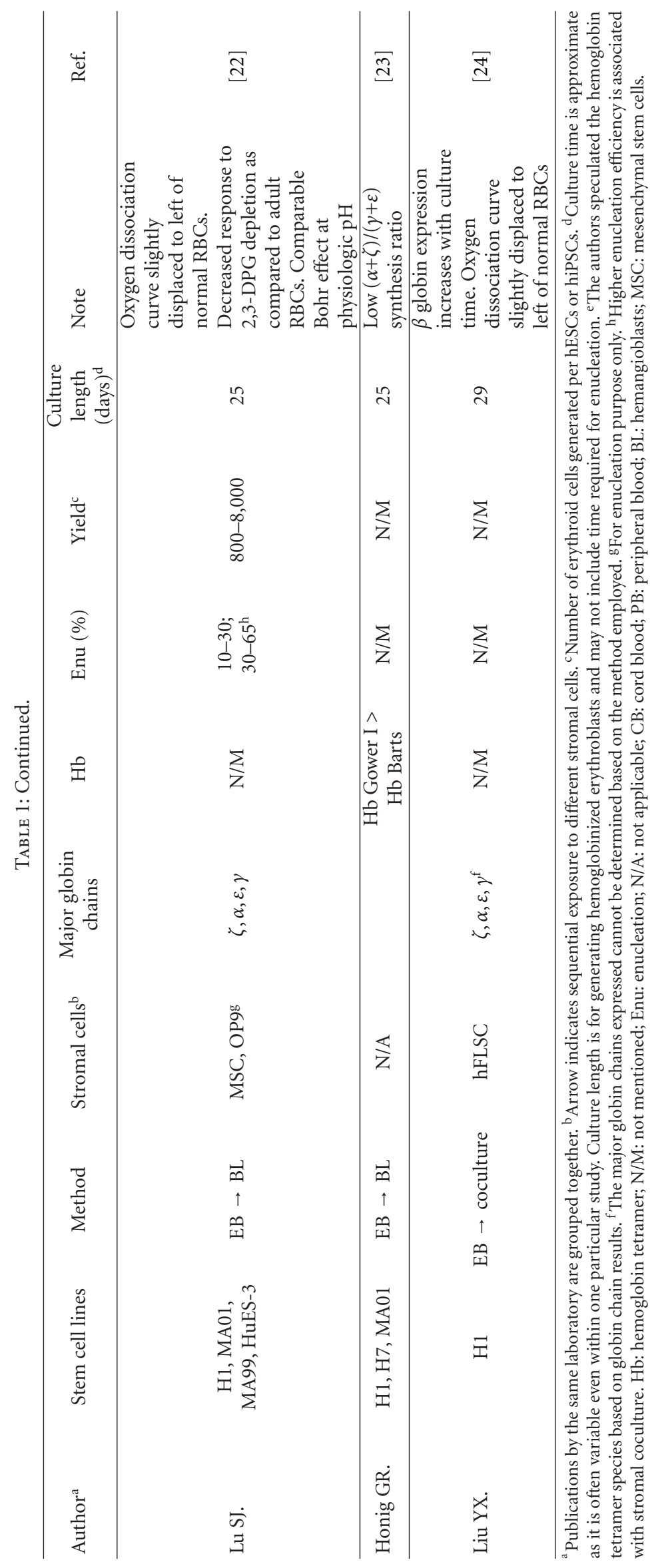


to note that care should be taken to avoid fusion among individual EBs as active migration of cells and the expression of transcription factor $\mathrm{Cd} \times 2$, known for its role in affecting cell fate decisions [34], have been observed in a subset of cells at the junction of fusing EBs [29]. While housing individual EBs in separate wells can prevent the aggregation, it is not recommended, as the paracrine effect enhances hematopoietic differentiation of EBs [33]. Encapsulating them in size-specified agarose capsules [35] may provide an alternative, although the hematopoietic differentiation of encapsulated EBs has not been fully examined.

An array of media has been used for the generation of EBs. Our laboratory has routinely used a medium essentially composed of Iscove's Modified Dulbecco's Media, fetal bovine serum (FBS), and protein-free hybridoma medium. Originally, we included basic fibroblast growth factor (bFGF) and vascular endothelial growth factor (VEGF) in the medium [17] and later found them to be unnecessary in FBS-supplemented media, although VEGF-165 has been reported to augment erythropoiesis during $\mathrm{EB}$ formation [16]. Lot specific effects of FBS on hematopoietic differentiation during EB formation have been noted and thus require prescreening of FBS for the EB assay (unpublished observations). Several laboratories have established serumfree media for EB formation, in which growth factor supplementation is required [26]. Bone morphogenetic protein 4 (BMP4), VEGF, granulocyte colony-stimulating factor (GCSF), interleukin-3 (IL-3), stem cell factor (SCF), Flt3 ligand, IL-6, insulin-like growth factor-2 (IGF-II), thrombopoietin (TPO), and bFGF have been used in an assortment of combinations to achieve induction of hematopoietic differentiation of EBs [22, 26, 30, 33, 36, 37]. A recent publication utilizing human plasma finds the addition of BMP4 and VEGF to be indispensable, consistent with the finding by Tian and colleagues [26], and the inclusion of SCF, TPO, Flt3 ligand, erythropoietin (EPO), IL-3, and IL-6 in the EB medium gives the optimal erythropoietic stimulation [20].

2.3. From EBs to Erythroid Cells. Different laboratories harvest EBs at different time points for further erythroid differentiation. Our protocol originally included an adherent culture step for erythroid induction, but was later streamlined to seeding dissociated day-7 EBs directly into a serum-free erythroid induction medium in which a large number of relatively pure erythroid cells $(>90 \%)$ can be obtained with 21 days of total culture time [17-19]. We find that EPO and SCF are absolutely required for the survival and differentiation of erythroid progenitors in EBs; IL-3 and IL-6 augment the expansion of erythroid cells, but G-CSF and GM-CSF are dispensable (unpublished data), similar to the conclusion of others [10]. If added, FBS has significant inhibitory effect on the growth and expansion of these erythroid progenitors from EBs, as previously noted by Zambidis et al. [21]. Furthermore, while dissociated day-7 EBs give rise to almost exclusively erythroid cells (>90\%) in the serum-free erythroid induction medium, day14 EBs generate a mixture of erythroid and myeloid cells [17]. Interestingly, while $\sim 5-10 \%$ cells of hESC line $\mathrm{H} 1-$ derived EBs express glycophorin-A between 7 and 14 days in our FBS-based system $[17,19]$, very little glycophorinA expression is detected in the H1-derived EBs up to 20 days in the human plasma-based cultures in spite of the hematopoietic cytokines supplementation in the medium [20]. The differences in culture time and cytokine supplements notwithstanding, Lapillonne et al. also find the unpurified, dissociated EBs from their human plasma-based cultures generating exclusively erythroid cells when placed in an erythroid expansion medium that also contains human plasma. Lu and colleagues take a different approach by placing dissociated day-3.5 EBs in blast-colony growth media with the addition of bFGF and recombinant tPTD-HoxB4 fusion protein to expand hemangioblasts prior to transfer into an erythroid differentiation and expansion culture [22]. A mixture of erythroid and nonerythroid cells is apparently obtained, which requires an overnight adhesion culture to remove nonerythroid cells.

\subsection{From Erythroblasts to RBCs. Extended ex vivo propaga-} tion of cells is associated with acquisition of genetic alterations, including transformation. Safety concerns about in vivo use of such cells therefore have been raised, specifically in the autologous setting. As terminally differentiated (and physiologically enucleated) cells, in this respect, erythrocytes possess considerable advantages over transplants of immature cells or cells with proliferative capacity in spite of their maturity, such as T cells. Several studies show that hESCand hiPSC-derived erythroblasts are capable of enucleation and become fully mature RBCs. A prolonged culture appears to be an important factor with or without the involvement of stromal cells. Qui and colleagues find enucleation can be obtained by sequential exposure of CD $34^{+}$cells sorted from FH-B-hTERT/H1 coculture to two sets of cytokines, followed by a 2-step 10-day coculture with MS-5 stromal cells [15]. The enucleation efficiency varies from $1.5 \%$ to $16 \%$ for the day-35 H1/FH-B-hTERT coculture, and $0 \%$ for the day-14 H1/FH-B-hTERT coculture. A 3-step culture that involves a 15-day coculture of H1/mFLSC coculture, followed by a 14-day erythroid burst colony formation, and then a 7-day liquid culture expansion also produces clusters of enucleated RBCs with enucleation efficiency ranging from $1.3 \%$ to $11.4 \%$ [13]. A higher degree of enucleation is achieved by $\mathrm{Lu}$ et al. with their hemangioblasts protocol composed of EB formation, hemangioblast expansion, sequential cytokine exposure of the blast cells, and then coculture with human mesenchymal stem cells (MSCs), with OP9 mouse stromal cells, or without stromal cells [22]. The enucleation efficiencies are 10\%-30\% without stromal cells, $\sim 30 \%$ on MSC stromal cells, and $30 \%-65 \%$ on OP9 cells, suggesting that the presence of stromal cells significantly facilitates the enucleation process. On the other hand, a recent finding by Lapillonne and colleagues shows that by supplementing culture media with human plasma, a significant degree of enucleation is attained without stromal cells in $\mathrm{H} 1$ hESCs-derived erythroid cells $(52 \%-66 \%)$ [20]. However, much lower degrees of enucleation (4\%-10\%) are obtained with erythroid cells derived from 2 separate hiPSC lines using the same protocol, suggesting that the reprogramming process or epigenetic memories retained, may impact the 
ability of hiPSC-derived erythroid cells to enucleate. Given the currently still incomplete efficiency of enucleation of erythroblasts and incomplete lineage determination in vitro and if these ex vivo generated erythroid cells were to be used in transfusion, it might be reasonable to filter them through conventional leukocyte filters, followed by irradiation with 3,000 cGy, as erythrocytes tolerate irradiation without critical loss of function.

2.5. Large-Scale Production of Erythroblasts or RBCs from hESCs or hiPSCs. Several publications have claimed that their respective protocol results in large-scale generations of erythroid cells from hESCs or hiPSCs, which is a prerequisite of any potential clinical usage. With the H1/FHB-hTERT coculture system, Qiu et al. obtain approximately 80 erythroid cells per hESC although it is estimated that a $50 \times 10^{3}$-fold expansion is achieved by a single CD $34^{+}$ cells from the coculture [15]. Using H1/mFLSC coculture, $\mathrm{Ma}$ and colleagues generate large erythroid bursts consisting of $\sim 0.2 \times 10^{6}$ erythroid cells per burst [13]. Taking into consideration the colony forming unit frequencies and further expansion potentials in liquid culture, roughly 1,200 erythroid cells are generated per hESC. A slightly higher production efficiency is achieved by using EB formation and human plasma supplementation that shows 1,500-3,500 erythroid cells are produced per hESC, but only 200-400 erythroid output per hiPSC [20]. Using recombinant tPTDHoxB4 fusion protein and hemangioblast approach, Lu and colleagues reported the generation of up to 8,000 erythroid cells per hESC [22]. The highest production efficiency is reported by Dias and colleagues employing the OP9 coculture system, followed by dissociation and reaggregation on low attachment plates with the addition of cytokines, and then coculture on MS-5 feeders [8]. Up to 200,000 erythroid cells are reported to be generated per hESC, and a similar level of expansion is also obtained with hiPSCs, although the requirement of murine cells for coculture throughout the differentiation procedure, in addition to the significantly longer culture time (70-120 days), remains a setback for potential clinical applications. Together, these data show that without significant progress in generating erythrocytes from hESCs or hiPSCs more cost effectively, the magnitude and cost of replacing donor-derived erythrocytes with these in vitro generated RBCs will be prohibitive, given that one RBC concentrate contains approximately $2 \times 10^{12}$ erythrocytes, that is, in a country like Germany, $8 \times 10^{18}\left(10^{11}\right.$ per head of the population) red blood cells are transfused in any given year.

\section{Characterization of hESC- and hiPSC-Derived Erythroid Cells}

3.1. Globin Chain Expression. Amongst all erythroidassociated characteristics, the globin expression phenotype has been studied most extensively in the hESC- and hiPSCderived erythroid cells. Our laboratory finds that the $\alpha$ globin, rather than embryonic $\zeta$ globin, is the major $\alpha$ locus globin expressed, although expression of $\zeta$ globin can be detected in all erythroid cells from $\mathrm{H} 1 \mathrm{hESC}$ at the protein level, suggesting a low, but pancellular expression of embryonic $\zeta$ globin [17], differing from the finding of Cerdan et al. that shows $\zeta$ globin is not expressed by hESCderived erythroblasts [16]. The expression of $\zeta$ globin is also confirmed by studies in erythroid cells generated from other hESC lines as well as H1 line [15, 21, 22, 24], and hiPSCs originated from either fetal or adult tissues [20]. We find the $\alpha / \zeta$ globin ratio does not change whether the erythroid cells are derived from day-7 or day-14 EBs [17], although Qiu and colleagues find that by extending the coculture time between hESCs and immortalized human fetal liver hepatocyte line FH-B-hTERT from 14 days to 35 days, a lineage switch occurs and these hESCs-derived erythroid cells express $\alpha$ locus globins with an increased $\alpha / \zeta$ globin ratio [15]. Furthermore, individual clonal erythroid expansion and maturation apparently is accompanied by a maturation switch characterized by an increasing $\alpha / \zeta$ globin ratio over time as the hESC-derived erythroid cells proceed from the stage of proerythroblasts to the stage of orthochromatic erythroblasts [15].

With the exception of the first two studies examining the hematopoietic/erythroid differentiation of hESC that claim the adult $\beta$ globin to be the major globin expressed by hESC-derived erythroid cells $[9,16]$, all other studies followed find embryonic $\varepsilon$ and fetal $\gamma$ globins to be the predominant $\beta$-locus globin expressed by these cells, at least initially, regardless of the differentiation methods employed or the ontogenic origin of the hiPSCs $[1,8,13,15,17-$ $22,24]$. While some studies find little changes in $\varepsilon / \gamma$ ratio in mature erythroid cells derived from different time points of culture [8, 17, 24], Qiu et al. reported that similar to their finding in the changes in $\alpha / \zeta$ globin ratio, increasing coculture time also leads to the generation of erythroid cells expressing mainly fetal $\gamma$ globin [15]. An increase in the adult $\beta$ globin expression with prolonged coculture time is also observed albeit its expression level is low ( $2 \%$ of all $\beta$-locus globins), consistent with the findings of others $[8,22,24]$. Interestingly, $\mathrm{Ma}$ et al. show a more drastic increase in $\beta$ globin expression with their erythroid cells derived from H1/mFLSC cells coculture. The globin expression in the erythroid cells of individual clones changes in a time-dependent manner: embryonic $\mathcal{E}$-globin-expressing erythroid cells from individual clones decrease from $100 \%$ to $\sim 50 \%$, whereas adult-type $\beta$-globin-expressing cells increase to $\sim 100 \%$ in all clones examined [13]. It is not known, however, whether the adult $\beta$ globin actually is the major $\beta$-locus globin expressed, given that $100 \%$ of the cells are also expressing $\gamma$ globin, and $\sim 50 \%$ of the cells are expressing $\varepsilon$ globin. Nevertheless, these studies provide supporting evidence that with prolonged coculture, and/or prolonged erythroid expansion, the erythroid cells adapt a more developmentally advanced globin expression pattern.

The transcriptional controls of the globin expression have not yet been studied in these cells, but a limited number of studies have been done to analyze the epigenetic landscape of $\beta$ globin locus of hESC-derived erythroid cells. It is found that domains of DNA hypomethylation spanning thousands of base pairs within domains of acetylated histones are 
established around the most highly expressed genes during each developmental stage when comparing hESC-derived erythroblasts to uncultured FL and bone marrow cells [38, 39]. In addition, our laboratory also finds that throughout development, human primary erythroblasts employ similar histone codes including enrichment of $\mathrm{H} 3 \mathrm{~K} 4 \mathrm{me} 3$ and selective depletion of $\mathrm{H} 3 \mathrm{~K} 27 \mathrm{me} 1$ to ensure proper temporal expression of specific globin genes (unpublished data). Furthermore, a looping mechanism is also at play to bring the hypersensitive sites of the LCR into physical contact with the $\varepsilon$ globin promoter, presumably to initiate transcription via the formation of an active chromatin hub, in the $\varepsilon$ globin producing hESC-derived erythroid cells.

3.2. Hemoglobin Analyses and Functionality. As compared to the globin chains, few studies have been conducted to study the hemoglobin tetramers in the hESC- or hiPSC-derived erythroid cells. Using CE-HPLC, it is found that the mature erythroid cells, derived from both hESCs and iPSCs based on the EB/human plasma culture system, express mostly $\mathrm{HbF}$ $(\alpha 2 \gamma 2)$ with little $\mathrm{Hb}$ Gower I $(\zeta 2 \varepsilon 2)$ or Gower II $(\alpha 2 \varepsilon 2)$ [20]. Furthermore, the CO-rebinding kinetics, which probe the allosteric equilibrium of $\mathrm{R}$ and $\mathrm{T}$ states, of hemoglobin from hESC- and hiPSC-derived erythroid cells, are almost identical to those of cord blood, with or without potent allosteric effector inositol hexaphosphate, suggesting that the $\mathrm{HbF}$ in these erythroid cells is functional [20]. In addition, the oxygen dissociation curve of hESC-derived erythroid cells is displaced to the left, similar to that of the human cord blood, as compared to that of adult RBCs, consistent with the higher affinity of $\mathrm{HbF}$ to oxygen than $\mathrm{HbA}[13$, $22,24]$. The decreased response to 2,3-diphosphoglycerate (2,3-DPG) depletion as compared to adult RBCs is consistent with the lack of interaction between HbF and 2,3-DPG [22]. In addition, hESC-derived erythroid cells also have high glucose-6-phosphate dehydrogenase activity, confirming that they are protected from oxidative damage [13]. Together, these data indicate that hESC- and hiPSC-derived erythroid cells will function as oxygen carriers like cord blood or fetal blood. In contrast, a recent study using the hemangioblast approach shows that hESC-derived erythroid cells produce mainly $\mathrm{Hb}$ Gower1 ( $\zeta 2 \varepsilon 2)$ with low levels of $\mathrm{HbF}, \mathrm{Hb}$ Portland $(\zeta 2 \gamma 2)$, and Hb Gower-2 ( $\alpha 2 \varepsilon 2)$, each represents less than $5 \%$ of the total hemoglobin [23]. Moreover, these hemangioblast-derived erythroid cells also have an elevated amount of $\mathrm{Hb}$ Barts ( ${ }^{\mathrm{G}} \gamma 4$ ) that accounts for $12 \%-18 \%$ of the total hemoglobin. As $\mathrm{Hb}$ Barts has extremely high affinity to oxygen, this finding appears to contradict the previous report by the same group that hESC-derived erythroid cells have an oxygen dissociation curve only slightly displaced to the left of the adult RBCs, and that they show a comparable Bohr effect at physiologic $\mathrm{pH}$ [22]. In addition to not releasing oxygen into the tissue due to extremely high oxygen affinity, $\mathrm{Hb}$ Barts is moderately insoluble, and therefore accumulates in the red blood cells. Hence, this study would argue that hESCderived erythroid cells, despite the large-scale production using hemangioblast protocol [22], can not be used for transfusion. The authors do offer that, however, with further maturation that leads to developmentally more advanced erythroblasts or enucleated RBCs, the level of Hb Barts may decrease in these hESC-derived erythroid cells.

\section{Future Challenges}

The magnitude and cost of replacing donor-derived erythrocytes with in vitro generated RBCs is prohibitive and requires significant progress in the expansion, maturation, and terminal differentiation/enucleation of erythrocytes to make the transfusion products cost effective. A recently published method of culturing hESCs and hiPSCs in suspension may aid in the scaling up production of hESCs and hiPSCs [40]. In addition, the proposed utilization of synthetic $3 \mathrm{D}$ structures mimicking bone marrow structure [41] and the manipulation of transcriptional environment, such as ectopic expression of engineered Nup98-HoxA10 fusion protein [42] and downregulation of microRNAs-126/125* [43], may improve the erythroid production efficiency.

However, even if a cost-effective method of generating erythrocytes from hESCs or hiPSCs were achieved, significant challenges remain to be met before the therapeutic use of these cells. While not expressing HLA, mature RBCs express many surface antigens against which regular (present in all individuals not expressing the antigen) or irregular (acquired after contact with the antigen) antibodies can be directed. Thirty blood group systems, or antigen families, with almost 400 antigens have been documented, and antibodies against all of these antigens have been described. If ignored, such antibodies cause acute, potentially deadly, or delayed hemolytic transfusion reactions. Therefore, the presence of such antibodies necessitates selection of antigennegative RBCs. Since antigens are expressed from genes located throughout the genome, any combination of blood group antigens can occur, thus in theory, an almost infinite number of hESC or iPSC lines would be required to generate compatible erythrocytes for all individuals. Generation of null variants for blood group antigens via genetic manipulation is not conducive as null variants have been described for many blood group antigens, but all of which are associated with structural defects and/or with the generation of alternative antigens, with the exception of $\mathrm{Rh} \mathrm{dd}$ ( $\mathrm{Rh}$-negative, the null variant which represents the counter antigen to $\mathrm{Rh} \mathrm{D}$ ) and $\mathrm{O}$ (absence of both $\mathrm{A}$ and $\mathrm{B}$ transferase). It may be conceptually reasonable to generate a single hiPSC line from an $\mathrm{O} \mathrm{Rh}$ negative ccddee $\mathrm{K}$ negative donor heterozygous for as many antigens as possible (to decrease foreign antigen dose and hence, antigenicity) and propagate it in huge biogenerators as a source for nonimmunized patients. For allo-(poly)sensitized or at risk, chronically transfusion-dependent patients, specifically those of rare blood types, autologous iPSC lines could be established, from which individualized erythrocytes could be generated and stored. It must be stressed, however, that the membrane protein and glycosylation profile of RBCs produced in vitro, even from autologous iPSC lines, may be altered compared to that of the in vivo produced RBCs, due to the lack of complex environment found in vivo, or an imbalanced transcription factor expression [44]. The important finding that iPSCs and their progeny are rejected in the autologous setting [45] 
appropriately brings to the forefront the issue of antigenicity of ex vivo generated cells due to expression of neoantigens. It may be plausible to enzymatically remove surface antigens. However, such approach has not been achieved except for A and B substances [46].

Furthermore, rigorous studies must be conducted to fully characterize these in vitro generated RBCs including their membrane surface potential, pliability, and half-life in vivo, in addition to profiling their hemoglobin packing, gas exchange properties, and immunogenicities. The sensitivity of the system is well illustrated by the recent evidence that has come forth about effects of ageing of erythrocytes on their therapeutic benefit-free hemoglobin, increased membrane rigidity, membrane damage, and altered $\mathrm{O}_{2}$ affinity of stored red cells have all been implicated in adverse outcomes observed with older erythrocytes [47]. Thus, even after all the challenges of devising suitable in vitro systems for expansion, maturation and terminal differentiation/enucleation of erythrocytes from primitive cell sources have been mastered, all of these biophysical parameters will still need to be carefully compared between in vitro and in vivo generated erythrocytes before they can be used clinically.

\section{Conclusions}

Significant progress has been made in generating erythroid cells from hESCs and hiPSCs. The production efficiency of up to 200,000 cells per hESC or iPSC has been achieved [8]. Unfortunately, the methodology that generates the highest number of erythroid cells per hESC is the least defined and relies heavily on the presence of murine stromal cells: first OP9, then MS-5. The hemoglobin expression pattern and enucleation efficiency are also less clear with erythroid cells derived from this particular method, than from the other mass production protocols [13, 20, 22, 23]. Further investigation of the hemoglobin tetramer composition is critical, as it is shown that erythroid cells generated from the protocol with the second-highest production efficiency (8,000 erythroid cells per hESC) have mostly Hb Gower I, followed by an elevated level of $\mathrm{Hb}$ Barts, which would not be suitable for transfusion [23]. The method proposed by Lapillonne and colleagues, without using animal products or stromal cell lines, generates moderate number of erythroid cells from hESCs (1,500-3,500 erythroid cells per hESC) that enucleate efficiently $(\sim 60 \%)$ with functional $\mathrm{HbF}$ tetramers as the major hemoglobin $(\sim 93 \%)$. Unfortunately, this method appears to work less efficiently with hiPSC, which is important since one of the major goals of generating RBCs is for the transfusion needs of patients with rare blood phenotypes or those that have been alloimmunized. The decreased efficiency of erythroid generation may be offset by prescreening for hiPSC lines with increased erythroid differentiation propensity, as line-to-line variation has been documented $[18,19]$, or by simply increasing the input number of hiPSCs. Alternatively, generating immortal erythroid progenitor lines capable of terminal differentiation and enucleation, which have been shown to be successful in murine ESC-derived cells [48], may be a viable option. Finally, little is known about these hESC- and hiPSC-derived erythroid cells other than their hemoglobin composition, gas exchange properties, and their growth and expansion pattern in response to various methods. Membrane compositions, surface antigen expression, immunogenicity, deformability, and half-life in vivo all require further and vigorous investigations before the transition from bench to bedside can occur.

\section{References}

[1] E. N. Olivier, C. Qiu, M. Velho, R. E. Hirsch, and E. E. Bouhassira, "Large-scale production of embryonic red blood cells from human embryonic stem cells," Experimental Hematology, vol. 34, no. 12, pp. 1635-1642, 2006.

[2] T. Peyrard, L. Bardiaux, C. Krause et al., "Banking of pluripotent adult stem cells as an unlimited source for red blood cell production: potential applications for alloimmunized patients and rare blood challenges," Transfusion Medicine Reviews, vol. 25, no. 3, pp. 206-216, 2011.

[3] J. A. Thomson, "Embryonic stem cell lines derived from human blastocysts," Science, vol. 282, no. 5391, pp. 1145-1147, 1998.

[4] J. Yu, M. A. Vodyanik, K. Smuga-Otto et al., "Induced pluripotent stem cell lines derived from human somatic cells," Science, vol. 318, no. 5858, pp. 1917-1920, 2007.

[5] K. Takahashi, K. Tanabe, M. Ohnuki et al., "Induction of pluripotent stem cells from adult human fibroblasts by defined factors," Cell, vol. 131, no. 5, pp. 861-872, 2007.

[6] H. Bonig, K. H. Chang, C. Geisen, E. Seifried, and C. Ware, "Blood types of current embryonic stem cell lines are not conducive to culturing "universal-donor" red blood cells," Transfusion, vol. 48, no. 5, pp. 1039-1040, 2008.

[7] A. Seifinejad, A. Taei, M. Totonchi et al., "Generation of human induced pluripotent stem cells from a Bombay individual: moving towards "universal-donor" red blood cells," Biochemical and Biophysical Research Communications, vol. 391, no. 1, pp. 329-334, 2010.

[8] J. Dias, M. Gumenyuk, and H. Kang, "Generation of red blood cells from human induced pluripotent stem cells," Stem Cells and Development, vol. 20, no. 9, pp. 1639-1647, 2011.

[9] D. S. Kaufman, E. T. Hanson, R. L. Lewis, R. Auerbach, and J. A. Thomson, "Hematopoietic colony-forming cells derived from human embryonic stem cells," Proceedings of the National Academy of Sciences of the United States of America, vol. 98, no. 19, pp. 10716-10721, 2001.

[10] O. Klimchenko, M. Mori, A. DiStefano et al., "A common bipotent progenitor generates the erythroid and megakaryocyte lineages in embryonic stem cell-derived primitive hematopoiesis," Blood, vol. 114, no. 8, pp. 1506-1517, 2009.

[11] K. Y. Lee, B. S. P. Fong, K. S. Tsang et al., "Fetal stromal niches enhance human embryonic stem cell-derived hematopoietic differentiation and globin switch," Stem Cells and Development, vol. 20, no. 1, pp. 31-38, 2011.

[12] M. H. Ledran, A. Krassowska, L. Armstrong et al., "Efficient hematopoietic differentiation of human embryonic stem cells on stromal cells derived from hematopoietic niches," Cell Stem Cell, vol. 3, no. 1, pp. 85-98, 2008.

[13] F. Ma, Y. Ebihara, K. Umeda et al., "Generation of functional erythrocytes from human embryonic stem cell-derived definitive hematopoiesis," Proceedings of the National Academy of Sciences of the United States of America, vol. 105, no. 35, pp. 13087-13092, 2008. 
[14] C. Qiu, E. Hanson, E. Olivier et al., "Differentiation of human embryonic stem cells into hematopoietic cells by coculture with human fetal liver cells recapitulates the globin switch that occurs early in development," Experimental Hematology, vol. 33, no. 12, pp. 1450-1458, 2005.

[15] C. Qiu, E. N. Olivier, M. Velho, and E. E. Bouhassira, "Globin switches in yolk sac-like primitive and fetal-like definitive red blood cells produced from human embryonic stem cells," Blood, vol. 111, no. 4, pp. 2400-2408, 2008.

[16] C. Cerdan, A. Rouleau, and M. Bhatia, "VEGF-A165 augments erythropoietic development from human embryonic stem cells," Blood, vol. 103, no. 7, pp. 2504-2512, 2004.

[17] K. H. Chang, A. M. Nelson, H. Cao et al., "Definitive-like erythroid cells derived from human embryonic stem cells coexpress high levels of embryonic and fetal globins with little or no adult globin," Blood, vol. 108, no. 5, pp. 1515-1523, 2006.

[18] K. H. Chang, A. Huang, R. K. Hirata, P. R. Wang, D. Russell, and T. Papayannopoulou, "Globin phenotype of erythroid cells derived from human induced pluripotent stem cells," Blood, vol. 115, no. 12, pp. 2553-2554, 2010.

[19] K.-H. Chang, A. M. Nelson, P. A. Fields et al., "Diverse hematopoietic potentials of five human embryonic stem cell lines," Experimental Cell Research, vol. 314, no. 16, pp. 29302940, 2008.

[20] H. Lapillonne, L. Kobari, C. Mazurier et al., "Red blood cell generation from human induced pluripotent stem cells: perspectives for transfusion medicine," Haematologica, vol. 95, no. 10, pp. 1651-1659, 2010.

[21] E. T. Zambidis, B. Peault, T. S. Park, F. Bunz, and C. I. Civin, "Hematopoietic differentiation of human embryonic stem cells progresses through sequential hematoendothelial, primitive, and definitive stages resembling human yolk sac development," Blood, vol. 106, no. 3, pp. 860-870, 2005.

[22] S. J. Lu, Q. Feng, J. S. Park et al., "Biologic properties and enucleation of red blood cells from human embryonic stem cells," Blood, vol. 112, no. 12, pp. 4475-4484, 2008.

[23] G. R. Honig, S. J. Lu, Q. Feng, L. N. Vida, B. S. Lee, and R. Lanza, " $\alpha$-thalassemia-like globin gene expression by primitive erythrocytes derived from human embryonic stem cells," Hemoglobin, vol. 34, no. 2, pp. 145-150, 2010.

[24] Y. X. Liu, W. Yue, L. Ji, X. Nan, and X. T. Pei, "Production of erythriod cells from human embryonic stem cells by fetal liver cell extract treatment," BMC Developmental Biology, vol. 10, article 85, 2010.

[25] G. Salvagiotto, S. Burton, C. A. Daigh, D. Rajesh, I. I. Slukvin, and N. J. Seay, "A defined, feeder-free, serum-free system to generate In Vitro hematopoietic progenitors and differentiated blood cells from hESCs and hiPSCs," PLoS One, vol. 6, no. 3, Article ID e17829, 2011.

[26] X. Tian, J. K. Morris, J. L. Linehan, and D. S. Kaufman, "Cytokine requirements differ for stroma and embryoid bodymediated hematopoiesis from human embryonic stem cells," Experimental Hematology, vol. 32, no. 10, pp. 1000-1009, 2004.

[27] M. A. Vodyanik, J. A. Bork, J. A. Thomson, and I. I. Slukvin, "Human embryonic stem cell-derived CD34+ cells: efficient production in the coculture with OP9 stromal cells and analysis of lymphohematopoietic potential," Blood, vol. 105, no. 2, pp. 617-626, 2005.

[28] D. ten Berge, W. Koole, C. Fuerer, M. Fish, E. Eroglu, and R. Nusse, "Wnt signaling mediates self-organization and axis formation in embryoid bodies," Cell Stem Cell, vol. 3, no. 5, pp. 508-518, 2008.
[29] M. D. Ungrin, C. Joshi, A. Nica, C. Bauwens, and P. W. Zandstra, "Reproducible, ultra high-throughput formation of multicellular organization from single cell suspension-derived human embryonic stem cell aggregates," PLoS One, vol. 3, no. 2, Article ID e1565, 2008.

[30] E. S. Ng, R. P. Davis, L. Azzola, E. G. Stanley, and A. G. Elefanty, "Forced aggregation of defined numbers of human embryonic stem cells into embryoid bodies fosters robust, reproducible hematopoietic differentiation," Blood, vol. 106, no. 5, pp. 1601-1603, 2005.

[31] P. W. Burridge, D. Anderson, H. Priddle et al., "Improved human embryonic stem cell embryoid body homogeneity and cardiomyocyte differentiation from a novel V-96 plate aggregation system highlights interline variability," Stem Cells, vol. 25, no. 4, pp. 929-938, 2007.

[32] K. Watanabe, M. Ueno, D. Kamiya et al., "A ROCK inhibitor permits survival of dissociated human embryonic stem cells," Nature Biotechnology, vol. 25, no. 6, pp. 681-686, 2007.

[33] S. H. Hong, T. Werbowetski-Ogilvie, V. Ramos-Mejia, J. B. Lee, and M. Bhatia, "Multiparameter comparisons of embryoid body differentiation toward human stem cell applications," Stem Cell Research, vol. 5, no. 2, pp. 120-130, 2010.

[34] H. Niwa, Y. Toyooka, D. Shimosato et al., "Interaction between Oct3/4 and Cdx2 determines trophectoderm differentiation," Cell, vol. 123, no. 5, pp. 917-929, 2005.

[35] S. M. Dang, S. Gerecht-Nir, J. Chen, J. Itskovitz-Eldor, and P. W. Zandstra, "Controlled, scalable embryonic stem cell differentiation culture," Stem Cells, vol. 22, no. 3, pp. 275-282, 2004.

[36] M. Pick, L. Azzola, A. Mossman, E. G. Stanley, and A. G. Elefanty, "Differentiation of human embryonic stem cells in serum-free medium reveals distinct roles for bone morphogenetic protein 4, vascular endothelial growth factor, stem cell factor, and fibroblast growth factor 2 in hematopoiesis," Stem Cells, vol. 25, no. 9, pp. 2206-2214, 2007.

[37] G. Lee, E. P. Papapetrou, H. Kim et al., "Modelling pathogenesis and treatment of familial dysautonomia using patientspecific iPSCs," Nature, vol. 461, no. 7262, pp. 402-406, 2009.

[38] M. Hsu, C. A. Richardson, E. Olivier et al., "Complex developmental patterns of histone modifications associated with the human $\beta$-globin switch in primary cells," Experimental Hematology, vol. 37, no. 7, pp. 799-806, 2009.

[39] M. J. Lathrop, M. Hsu, C. A. Richardson et al., "Developmentally regulated extended domains of DNA hypomethylation encompass highly transcribed genes of the human $\beta$-globin locus," Experimental Hematology, vol. 37, no. 7, pp. 807-813, 2009.

[40] M. Amit, J. Chebath, V. Margulets et al., "Suspension culture of undifferentiated human embryonic and induced pluripotent stem cells," Stem Cell Reviews and Reports, vol. 6, no. 2, pp. 248-259, 2010.

[41] D. J. Anstee, "Production of erythroid cells from human embryonic stem cells (hESC) and human induced pluripotent stem cells (hiPS)," Transfusion Clinique et Biologique, vol. 17, no. 3, pp. 104-109, 2010.

[42] J. Ji, R. M. Risueño, S. Hong et al., "Brief report: ectopic expression of Nup98-HoxA10 augments erythroid differentiation of human embryonic stem cells," Stem Cells, vol. 29, no. 4, pp. 736-741, 2011.

[43] X. Huang, E. Gschweng, B. Van Handel, D. Cheng, H. K. A. Mikkola, and O. N. Witte, "Regulated expression of microRNAs-126/126* inhibits erythropoiesis from human embryonic stem cells," Blood, vol. 117, no. 7, pp. 2157-2165, 2011. 
[44] B. K. Singleton, N. M. Burton, C. Green, R. L. Brady, and D. J. Anstee, "Mutations in EKLFIKLF1 form the molecular basis of the rare blood group $\operatorname{In}(\mathrm{Lu})$ phenotype," Blood, vol. 112, no. 5, pp. 2081-2088, 2008.

[45] T. Zhao, Z.-N. Zhang, Z. Rong, and Y. Xu, "Immunogenicity of induced pluripotent stem cells," Nature, vol. 474, no. 7350, pp. 212-216, 2011.

[46] Q. P. Liu, G. Sulzenbacher, H. Yuan et al., "Bacterial glycosidases for the production of universal red blood cells," Nature Biotechnology, vol. 25, no. 4, pp. 454-464, 2007.

[47] G. J. C. G. M. Bosman, J. M. Werre, F. L. A. Willekens, and V. M. J. Novotný, "Erythrocyte ageing in vivo and in vitro: structural aspects and implications for transfusion," Transfusion Medicine, vol. 18, no. 6, pp. 335-347, 2008.

[48] Y. Nakamura, T. Hiroyama, K. Miharada, and R. Kurita, "Red blood cell production from immortalized progenitor cell line," International Journal of Hematology, vol. 93, no. 1, pp. 5-9, 2010. 

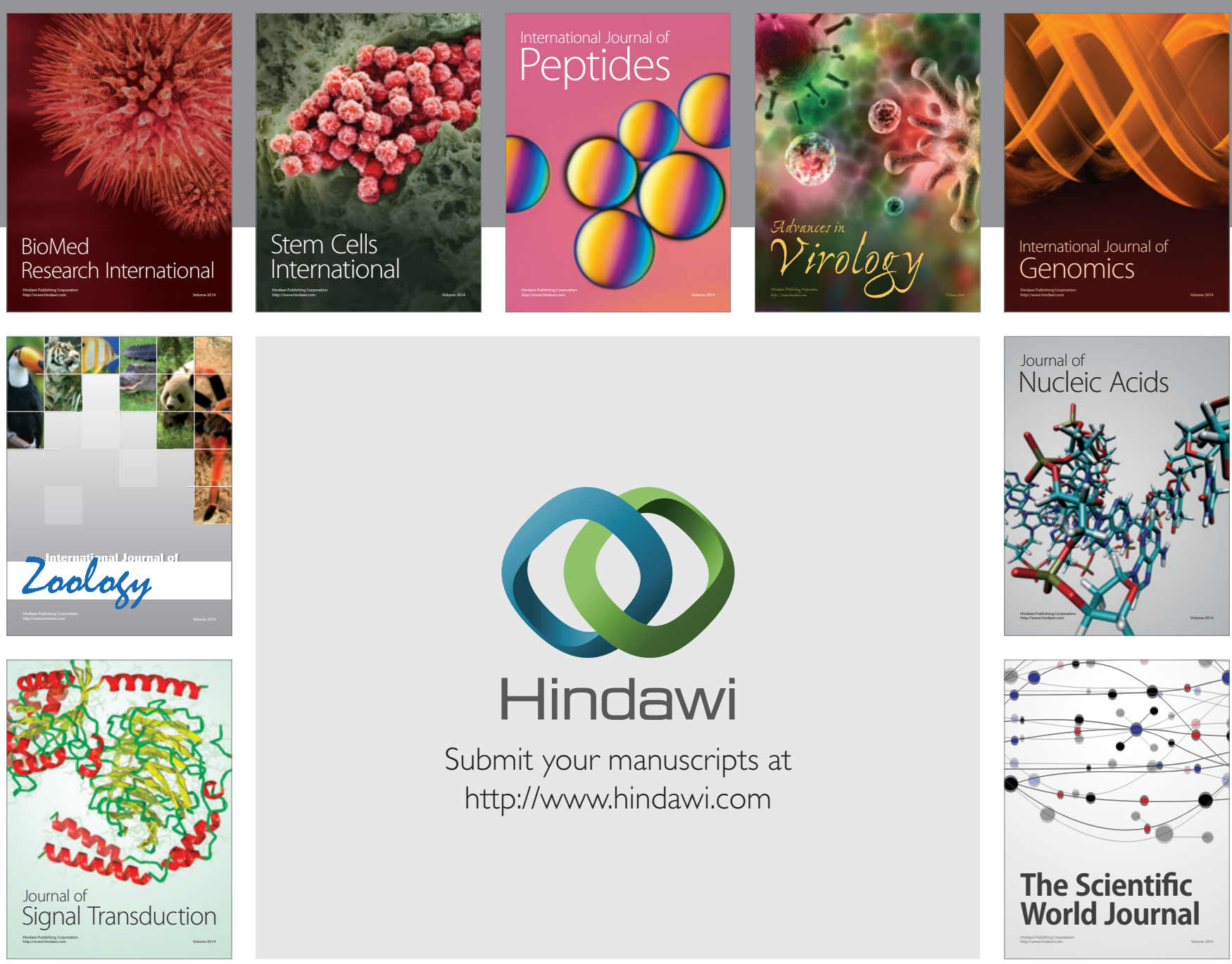

Submit your manuscripts at

http://www.hindawi.com
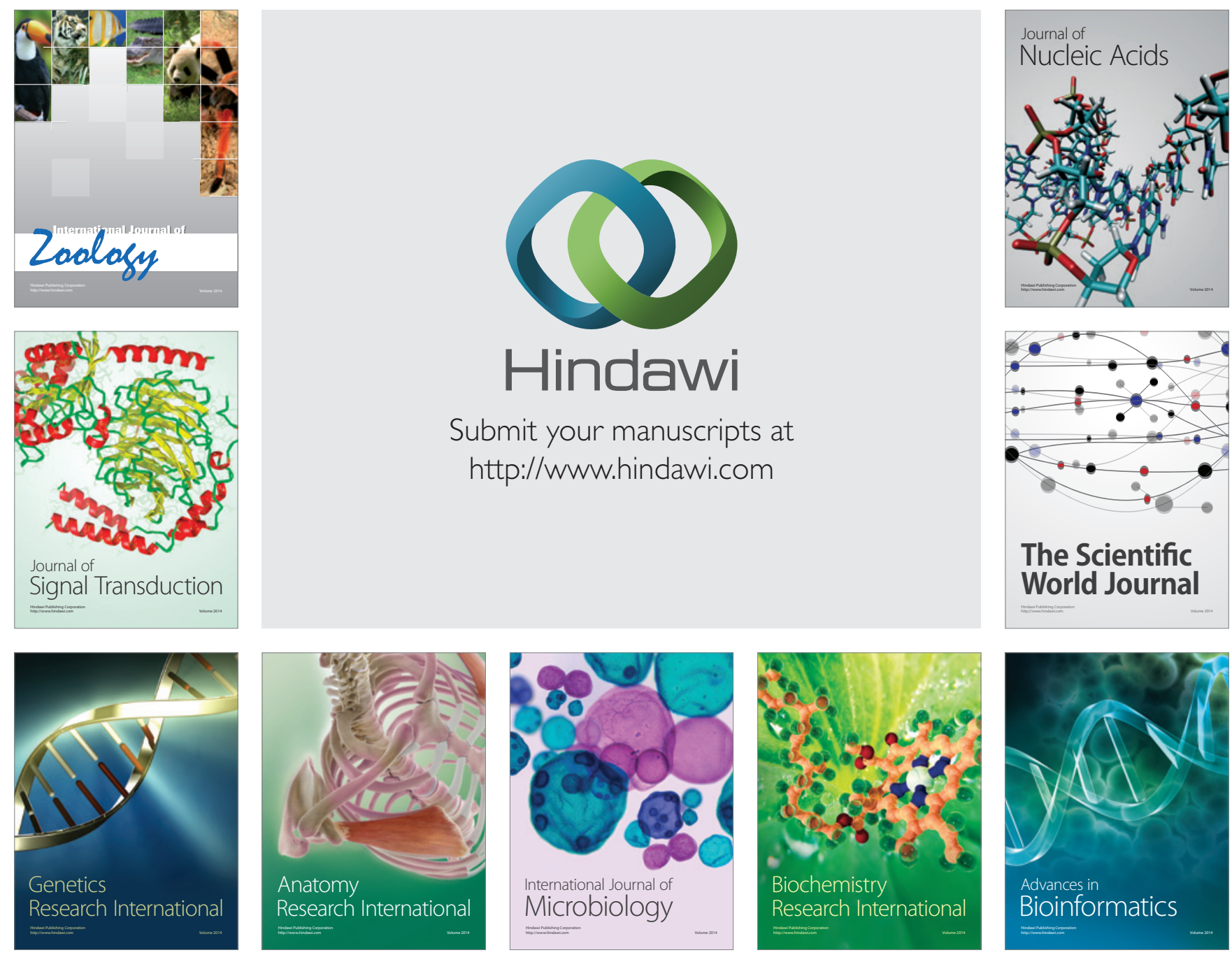

The Scientific World Journal
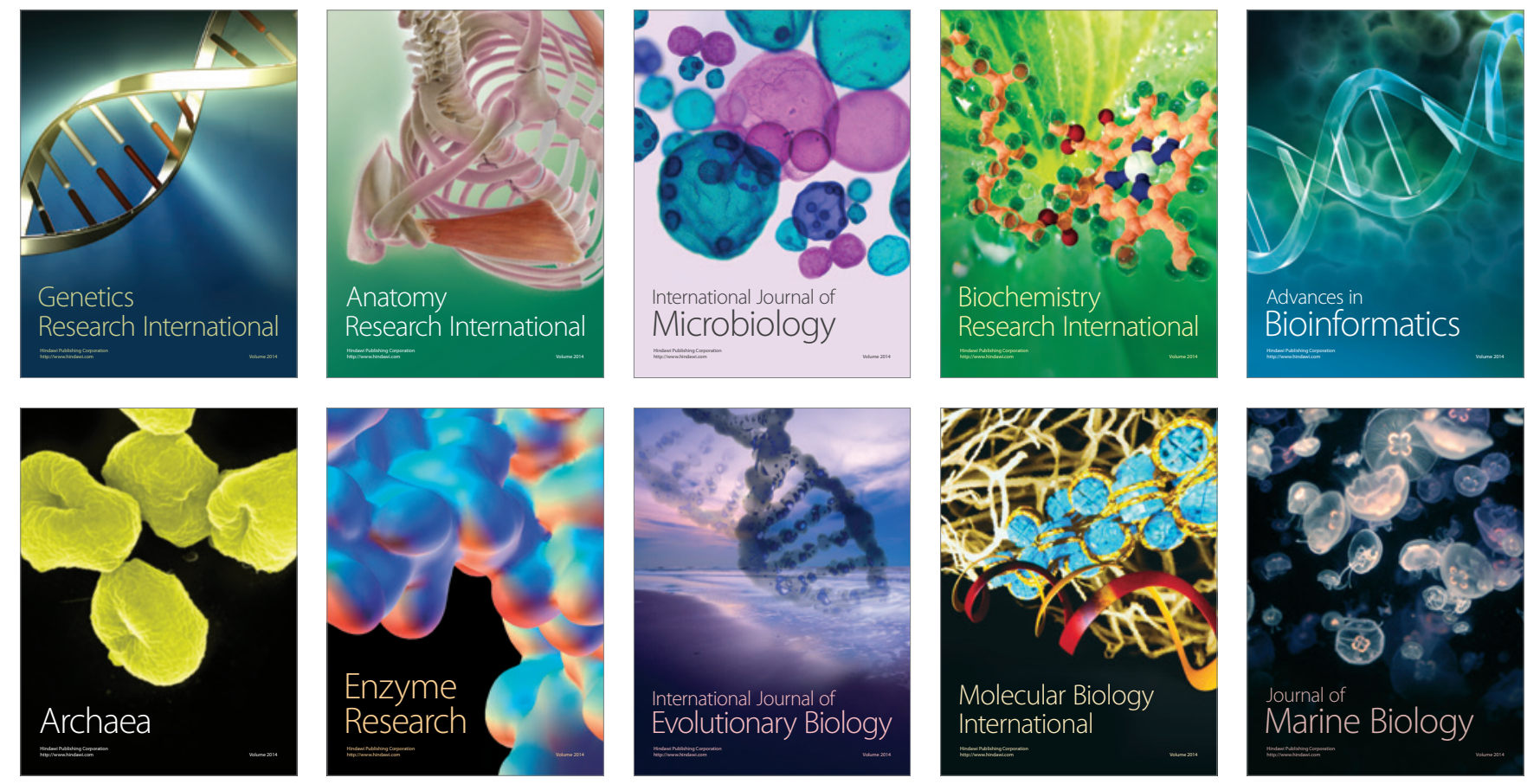